\title{
Numerical solution for a new fuzzy transform of hyperbolic goursat partial differential equation
}

\author{
Nur Syazana Saharizan, Nurnadiah Zamri \\ Faculty of Informatics and Computing, Universiti Sultan Zainal Abidin, Malaysia
}

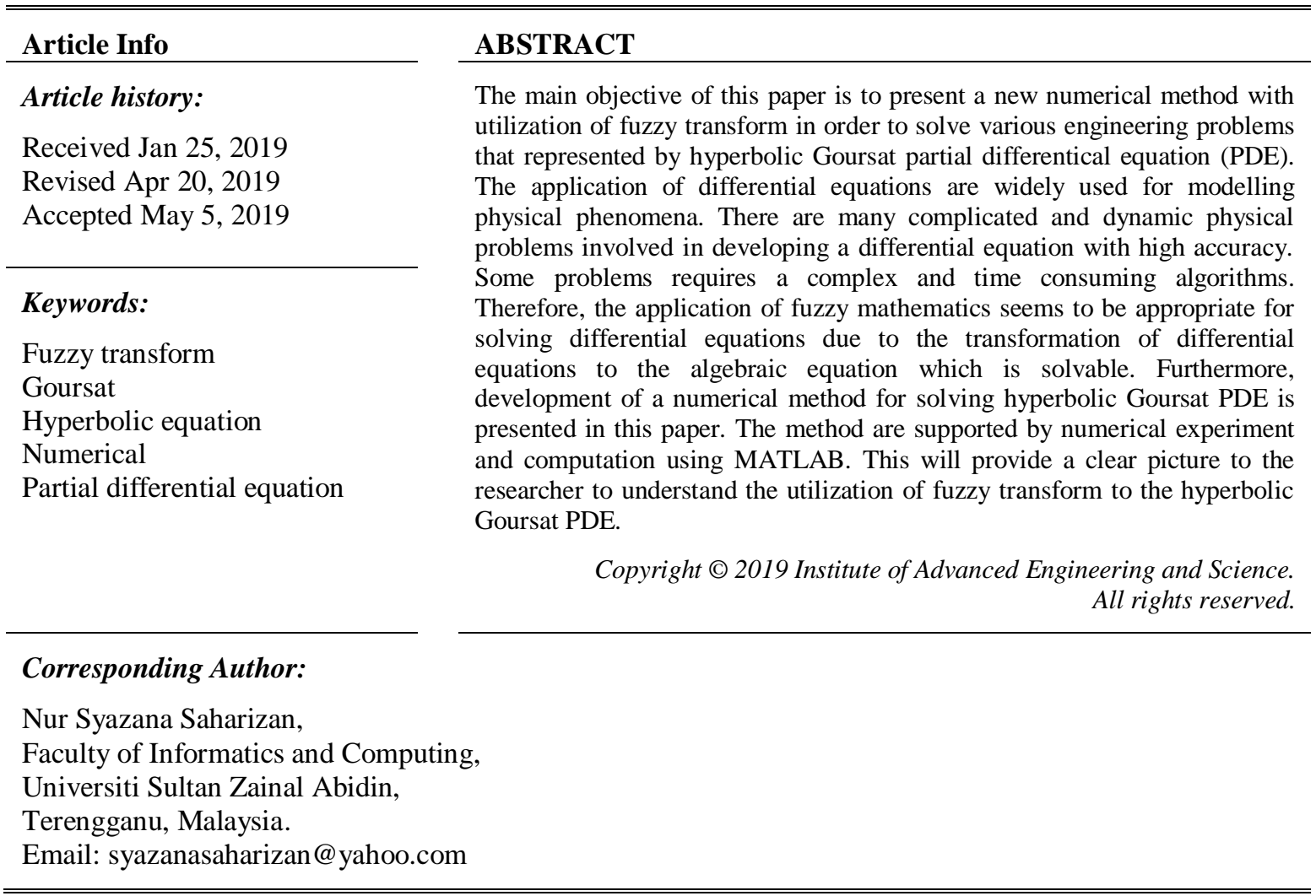

\section{INTRODUCTION}

Recently, various techniques and method have been developed and proposed for the numerical solution of ordinary differential equations and partial differential equations. The hyperbolic Goursat PDE arises in linear and nonlinear equations involving mixed derivatives. Researchers embark on developing various numerical methods to solve the problem such as Runge Kutta method [1], Two Dimensional Differential Transform Method [2], Finite Difference Method [3, 4], Variational Iteration Method [5] Adomian's Decomposition Method [6] and Explicit Numerical Scheme [7].

Fuzzy transform has been utilized and introduced as an approximation method in order to solve various differential equation including ordinary differential equation and partial differential equation [8-15]. It has been demonstrated that if the functions are replaced by an approximation scheme, at some point where complex computation appear, it can be simplified by fuzzy approximation method.

The main idea of this paper is to apply the fuzzy transform at both sides of Goursat hyperbolic PDE and approximate the functions using finite difference method and arithmetic mean.

\section{PRELIMINARIES}

This section is divided into two subsections, which are fuzzy transform and hyperbolic Goursat partial differential equation. 


\subsection{The fuzzy transform}

Fuzzy transform has been introduced by Irina Perfiliev [16]. The Fuzzy Set Theory concept by Lotfi Zadeh [17] is the fundamental of development of fuzzy transform. Some basic definitions is included to demonstrate the Fuzzy Transform.

Definition 1 Let $x_{i}=a+h(i-1)$ be nodes on $[a, b]$ where $h=\frac{b-a}{n-1}, n \geq 2$ and $i=1, \ldots, n$. We say that fuzzy sets $A_{1}(x), \ldots, A_{n}(x)$ defined on $[a, b]$ are basic functions and form a fuzzy partition of $[a, b]$ if the following conditions hold true for each $i=1, \ldots, n$ :

$A_{i}:[a, b] \rightarrow[0,1], A_{i}\left(x_{i}\right)=1$,

$A_{i}(x)=0_{\text {if }} x \notin\left(x_{i-1}, x_{i+1}\right)_{\text {where }} x_{0}=a, x_{n+1}=b$,

$A_{i}(x)$ is continuous,

$A_{i}(x)$ is strictly increases on $\left[x_{i-1}, x_{i}\right]$ and strictly decrease on $\left[x_{i}, x_{i+1}\right]$,

$\sum_{i=1}^{n} A_{i}(x)=1$, for all $x \in[a, b]$,

$A_{i}\left(x_{i}-x\right)=A_{i}\left(x_{i}+x\right)$, for all $x \in[0, h], i=2, \ldots, n-1, n>2$,

$A_{i+1}(x)=A_{i}(x-h), f$ or all $x \in[a+h, b], i=2, \ldots, n-2, n>2$,

Definition 2 Let $A_{1}(x), \ldots, A_{n}(x)$ be basic functions which form a fuzzy partition on $[a, b]$. The n-tuple of real numbers $\left[F_{1}, \ldots, F_{n}\right]$ given by

$$
F_{i}=\frac{\int_{a}^{b} f(x) A_{i}(x) d x}{\int_{a}^{b} A_{i}(x) d x}, i=1, \ldots, n
$$

is the direct fuzzy transform of function $\mathrm{f}$ with respect to $A_{1}(x), \ldots, A_{n}(x)$. The $\left[F_{1}, \ldots, F_{n}\right]$ are called the component of Fuzzy Transform.

Definition 3 Let the $\left[F_{1}, \ldots, F_{n}\right]$ be the direct Fuzzy Transform of function $\mathrm{f}$ with respect to

$$
\begin{aligned}
& A_{1}(x), \ldots, A_{n}(x) . \text { Hence, the function } \\
& f_{n}^{F}(x)=\sum_{i=1}^{n} F_{i} A_{i}(x)
\end{aligned}
$$

is called the inverse Fuzzy Transform of $\mathrm{f}$.

Definition 4 Let $f(x, y)$ be an arbitrary continuous function on $D=[a, b] \times[c, d]$ and real matrix $F^{2}[f]=\left[F_{i j}\right]_{n \times m}$ given by

$$
F_{i j}=\frac{\int_{c}^{d} \int_{a}^{b} f(x, y) A_{i}(x) B_{j}(y) d x d y}{\int_{c}^{d} \int_{a}^{b} A_{i}(x) B_{j}(y) d x d y}, i=1, \ldots, n, \quad j=1, \ldots, m
$$

is the Fuzzy Transform of $\mathrm{f}$ with respect to the given fuzzy partition. The reals $F_{i j}$ are the components of the Fuzzy Transform of $f$. In the next section, a brief explanation on the hyperbolic Goursat partial differential equation is discussed. 


\subsection{Hyperbolic goursat partial differential equation}

The Goursat equation is a second order hyperbolic PDE involving two independent variables $x$ and $y$. The general form of the Goursat problem is given by Wazwaz [18].

$$
\begin{aligned}
& \frac{\partial^{2} u(x, y)}{\partial x \partial y}=g\left(x, y, u, u_{x}, u_{y}\right), \\
& u(x, 0)=\phi(x), \\
& u(0, y)=\psi(y), \\
& \phi(0)=\psi(0)=u(0,0) \\
& 0 \leq x \leq a, \quad 0 \leq y \leq b
\end{aligned}
$$

where $\frac{\partial^{2} u(x, y)}{\partial x \partial y}$ is the mixed derivative in space $x$ and $y$ while the term $g\left(x, y, u, u_{x}, u_{y}\right)$ is a function consists of a combination of the independent variables $x$ and $y$, the dependent variable $u$ and the derivative terms $u_{x}$ and $u_{y}$.

The Goursat problem arises in linear hyperbolic PDE and nonlinear hyperbolic PDE. A linear Goursat PDE necessarily satisfies two particular conditions otherwise, it will be considered as a nonlinear equation. The first condition is the power of the dependent variable $u$ and the derivative term $u_{x}$ and $u_{y}$ must be one [18]. Besides, the coefficients of the dependent variable $u$ and the derivative term $u_{x}$ and $u_{y}$ must be the constant or the independent variables $x$ and $y$ [18]. In this paper, a Goursat PDE will be experimented. Some source of errors might occur during handling the Goursat PDE. The round off error usually occurs while allocating with the computing software because of its inability to deal with certain numbers. The terms must be approximated by using any certain suitable method where the truncation error might take place as well. Every approximation at certain grid points will be computed by using a computing software. The propagation error might occur since the approximation from present iteration depends on the approximation from the previous iteration and so forth. Hence, several difficulties in discretizing the Goursat equation with the derivative term may arise. Thus, it is a need to propose a new method on hyperbolic Goursat partial differential equation. This new method is discussed in the next section.

\section{THE PROPOSED OF FUZZY TRANSFORM METHOD FOR HYPERBOLIC GOURSAT PARTIAL DIFFERENTIAL EQUATION}

In this section, a new numerical method involving fuzzy transform is developed. Certain simplification of complex computations could be achieved by replacing the original function with an approximation model [8]. The main idea is to apply the fuzzy transform to both sides of the hyperbolic Goursat PDE. The application of fuzzy transform to the hyperbolic Goursat PDE lead to the transformation from differential equation to the algebraic equation which is solvable. The aid from MATLAB is very beneficial in calculating the exact solution and approximate solution at each grid point as well as the relative errors and the average. The relative error is used to describe the accuracy of the numerical method. The proposed numerical method can be extended in solving differential equation involving more variables.

Let the domain $D^{2}$ be given by a Cartesian product of two real intervals $X=[0,1]$ and $Y=[0, R]$ Let $u(x, y)$ be a continuous solution of the following hyperbolic Goursat partial differential equation.

$$
\frac{\partial^{2} u(x, y)}{\partial x \partial y}=g\left(x, y, u, u_{x}, u_{y}\right)
$$

After applying the fuzzy transform, the hyperbolic Goursat PDE is transferred to the following algebraic equation: 


$$
F^{2}\left[u^{x y}\right]=F^{2}[g]
$$

Where:

$$
F^{2}\left[u^{x y}\right]=\left[\begin{array}{cccc}
U_{11}^{x y} & U_{12}^{x y} & \cdots & U_{1 m}^{x y} \\
U_{21}^{x y} & U_{22}^{x y} & \cdots & U_{2 m}^{x y} \\
\vdots & \ddots & \ddots & \vdots \\
\vdots & \cdots & \cdots & \vdots \\
U_{n 1}^{x y} & \cdots & \cdots & U_{n m}^{x y}
\end{array}\right]
$$

is the matrix of the fuzzy transform components of $\frac{\partial^{2} u(x, y)}{\partial x \partial y}$, and components of $g$ represented by matrix of the fuzzy transform as below:

$$
F^{2}[g]=\left[\begin{array}{cccc}
G_{11} & G_{12} & \cdots & G_{1 m} \\
G_{21} & G_{21} & \cdots & G_{2 m} \\
\vdots & \ddots & \ddots & \vdots \\
\vdots & \cdots & \cdots & \vdots \\
G_{n 1} & \cdots & \cdots & G_{n m}
\end{array}\right]
$$

Since the partial differential equation $\frac{\partial^{2} u(x, y)}{\partial x \partial y}$ is unknown, the partial derivative is replaced by the forward finite difference approximation.

$$
\begin{aligned}
& \frac{\partial^{2} u(x, y)}{\partial x \partial y} \text { is replaced by } \\
& \frac{1}{h^{x} h^{y}}\left[u\left(x+h^{x}, y+h^{y}\right)-u\left(x+h^{x}, y\right)-u\left(x, y+h^{y}\right)+u(x, y)\right]
\end{aligned}
$$

Next, the approximation of $U_{i j}^{x y}$ is as follows:

$$
\begin{aligned}
U_{i j}^{x y} & =\frac{\int_{c}^{d} \int_{a}^{b} \frac{\partial^{2} u(x, y)}{\partial x \partial y} A_{i}(x) B_{j}(y) d x d y}{\int_{c}^{d} \int_{a}^{b} A_{i}(x) B_{j}(y) d x d y} \\
& \approx \frac{\int_{c}^{d} \int_{a}^{b}\left(\frac{1}{h^{x} h^{y}}\left[u\left(x+h^{x}, y+h^{y}\right)-u\left(x+h^{x}, y\right)-u\left(x, y+h^{y}\right)+u(x, y)\right]\right) A_{i}(x) B_{j}(y) d x d y}{\int_{c}^{d} \int_{a}^{b} A_{i}(x) B_{j}(y) d x d y}
\end{aligned}
$$




$$
\begin{aligned}
& =\frac{1}{h^{x} h^{y}}\left[\begin{array}{c}
\frac{\int_{c}^{d} \int_{a}^{b}\left(u\left(x+h^{x}, y+h^{y}\right)\right) A_{i}(x) B_{j}(y) d x d y}{\int_{c}^{d} \int_{a}^{b} A_{i}(x) B_{j}(y) d x d y}-\frac{\int_{c}^{d} \int_{a}^{b}\left(u\left(x+h^{x}, y\right)\right) A_{i}(x) B_{j}(y) d x d y}{\int_{c}^{d} \int_{a}^{b} A_{i}(x) B_{j}(y) d x d y} \\
-\frac{\int_{c}^{d} \int_{a}^{b}\left(u\left(x, y+h^{y}\right)\right) A_{i}(x) B_{j}(y) d x d y}{\int_{c}^{d} \int_{a}^{b} A_{i}(x) B_{j}(y) d x d y}+\frac{\int_{c}^{d} \int_{a}^{b}(u(x, y)) A_{i}(x) B_{j}(y) d x d y}{\int_{c}^{d} \int_{a}^{b} A_{i}(x) B_{j}(y) d x d y}
\end{array}\right] \\
& =\frac{1}{h^{x} h^{y}}\left(U_{(i+1)(j+1)}-U_{(i+1) j}-U_{i(j+1)}+U_{i, j}\right)
\end{aligned}
$$

In order to produce more accurate approximation of $G_{i j}$, an arithmetic mean technique has been utilized. The approximation is as below:

$$
\begin{aligned}
& g\left(x, y, u, u_{x}, u_{y}\right) \text { is replaced by } \\
& \frac{1}{4}\left[g\left(x+h^{x}, y+h^{y}\right)+g(x, y)+g\left(x+h^{x}, y\right)+g\left(x, y+h^{y}\right)\right] \\
& G_{i j}=\frac{\int_{c}^{d} \int_{a}^{b} g\left(x, y, u, u_{x}, u_{y}\right) A_{i}(x) B_{j}(y) d x d y}{\int_{c}^{d} \int_{a}^{b} A_{i}(x) B_{j}(y) d x d y} \\
& \approx \frac{\int_{c}^{d} \int_{a}^{b}\left(\frac{1}{4}\left[g\left(x+h^{x}, y+h^{y}\right)+g(x, y)+g\left(x+h^{x}, y\right)+g\left(x, y+h^{y}\right)\right]\right) A_{i}(x) B_{j}(y) d x d y}{\int_{c}^{d} \int_{a}^{b} A_{i}(x) B_{j}(y) d x d y} \\
& =\frac{1}{4}\left[\begin{array}{c}
\frac{\int_{c}^{d} \int_{a}^{b}\left(g\left(x+h^{x}, y+h^{y}\right)\right) A_{i}(x) B_{j}(y) d x d y}{\int_{c}^{d} \int_{a}^{b} A_{i}(x) B_{j}(y) d x d y}+\frac{\int_{c}^{d} \int_{a}^{b}(g(x, y)) A_{i}(x) B_{j}(y) d x d y}{\int_{c}^{d} \int_{a}^{b} A_{i}(x) B_{j}(y) d x d y} \\
+\frac{\int_{c}^{d} \int_{a}^{b}\left(g\left(x+h^{x}, y\right)\right) A_{i}(x) B_{j}(y) d x d y}{\int_{c}^{d} \int_{a}^{b} A_{i}(x) B_{j}(y) d x d y}+\frac{\int_{c}^{d} \int_{a}^{b}\left(g\left(x, y+h^{y}\right)\right) A_{i}(x) B_{j}(y) d x d y}{\int_{c}^{d} \int_{a}^{b} A_{i}(x) B_{j}(y) d x d y}
\end{array}\right] \\
& =\frac{1}{4}\left(G_{(i+1)(j+1)}+G_{i, j}+G_{(i+1), j}+G_{i(j+1)}\right)
\end{aligned}
$$

Thus, the recursive equation is as below

$$
\frac{1}{h^{x} h^{y}}\left(U_{(i+1)(j+1)}-U_{(i+1) j}-U_{i(j+1)}+U_{i, j}\right)=\frac{1}{4}\left(G_{(i+1)(j+1)}+G_{i, j}+G_{(i+1), j}+G_{i(j+1)}\right)
$$

\section{NUMERICAL EXPERIMENT}

Consider the following hyperbolic Goursat PDE

$$
\begin{aligned}
& u_{x y}=u, \\
& u(x, 0)=e^{x}, \\
& u(0, y)=e^{y}, \\
& 0 \leq x \leq 4, \quad 0 \leq \mathrm{y} \leq 4 .
\end{aligned}
$$


The exact analytical solution of the Goursat problem is $u(x, y)=e^{x+y}$ [19]. The accuracy of the method is investigated by observing the relative error and average relative error at different values of step size, $h$ as shown in Table 1, Table 2 and Table 3.

Table 1. Approximate Solutions at Various Grid Points and Step Size, h

\begin{tabular}{cccccc}
\hline$U(x, y)$ & Exact solution & 0.0025 & 0.005 & 0.025 & 0.05 \\
\hline$U(0.25,0.25)$ & 1.6487213 & 1.6487214 & 1.6487216 & 1.6487298 & 1.6487554 \\
$U(0.50,0.50)$ & 2.7182818 & 2.7182823 & 2.7182837 & 2.7183280 & 2.7184667 \\
$U(0.75,0.75)$ & 4.4816891 & 4.4816905 & 4.4816949 & 4.4818339 & 4.4822684 \\
$U(1.00,1.00)$ & 7.3890561 & 7.3890598 & 7.3890708 & 7.3894227 & 7.3905227 \\
$U(1.25,1.25)$ & 12.1824940 & 12.1825023 & 12.1825272 & 12.1833242 & 12.1858159 \\
$U(1.50,1.50)$ & 20.0855369 & 20.0855545 & 20.0856072 & 20.0872951 & 20.0925718 \\
$U(1.75,1.75)$ & 33.1154520 & 33.1154876 & 33.1155944 & 33.1190128 & 33.1296999 \\
$U(2.00,2.00)$ & 54.5981500 & 54.5982199 & 54.5984295 & 54.6051377 & 54.6261111 \\
\hline
\end{tabular}

Table 2. Relative errors, $E_{i, j}(h)$ with $\mathrm{h}=0.0025$

\begin{tabular}{ccccc}
\hline$y$ & & & $x$ & \\
& 0.5 & 1.0 & 1.5 & 2.0 \\
\hline 0.5 & $1.6996495 \mathrm{e}-07$ & $2.8515340 \mathrm{e}-07$ & $3.6294990 \mathrm{e}-07$ & $4.1532779 \mathrm{e}-07$ \\
1.0 & $2.8515340 \mathrm{e}-07$ & $4.9606933 \mathrm{e}-07$ & $6.5061317 \mathrm{e}-07$ & $7.6293317 \mathrm{e}-07$ \\
1.5 & $3.6294990 \mathrm{e}-07$ & $6.5061317 \mathrm{e}-07$ & $8.7527807 \mathrm{e}-07$ & $1.0485725 \mathrm{e}-06$ \\
2.0 & $4.1532779 \mathrm{e}-07$ & $7.6293317 \mathrm{e}-07$ & $1.0485725 \mathrm{e}-06$ & $1.2796936 \mathrm{e}-06$ \\
\hline
\end{tabular}

Table 3. Average relative errors, $\bar{E}(h)$ with several step size, $\mathrm{h}$

\begin{tabular}{cc}
\hline Step size, $h$ & Average relative error, $\bar{E}(h)$ \\
\hline 0.0025 & $4.2688467 \mathrm{e}-07$ \\
0.005 & $1.7111414 \mathrm{e}-06$ \\
0.025 & $4.3506679 \mathrm{e}-05$ \\
0.05 & $1.7771664 \mathrm{e}-04$ \\
\hline
\end{tabular}

It indicate that the relative errors become smaller and approaching zero as the step size, $h$ tends to zero. Hence, the accuracy of the method point out to be improved as the step size, $h$ decreasing. For a better understanding, the average relative errors, $\bar{E}(h)$ for the step size $\mathrm{h}=0.0025, \mathrm{~h}=0.005, \mathrm{~h}=0.025$ and $\mathrm{h}=0.05$ are shown in Table 3. Figure 1 shows the graph of exact solution and approximate solution.
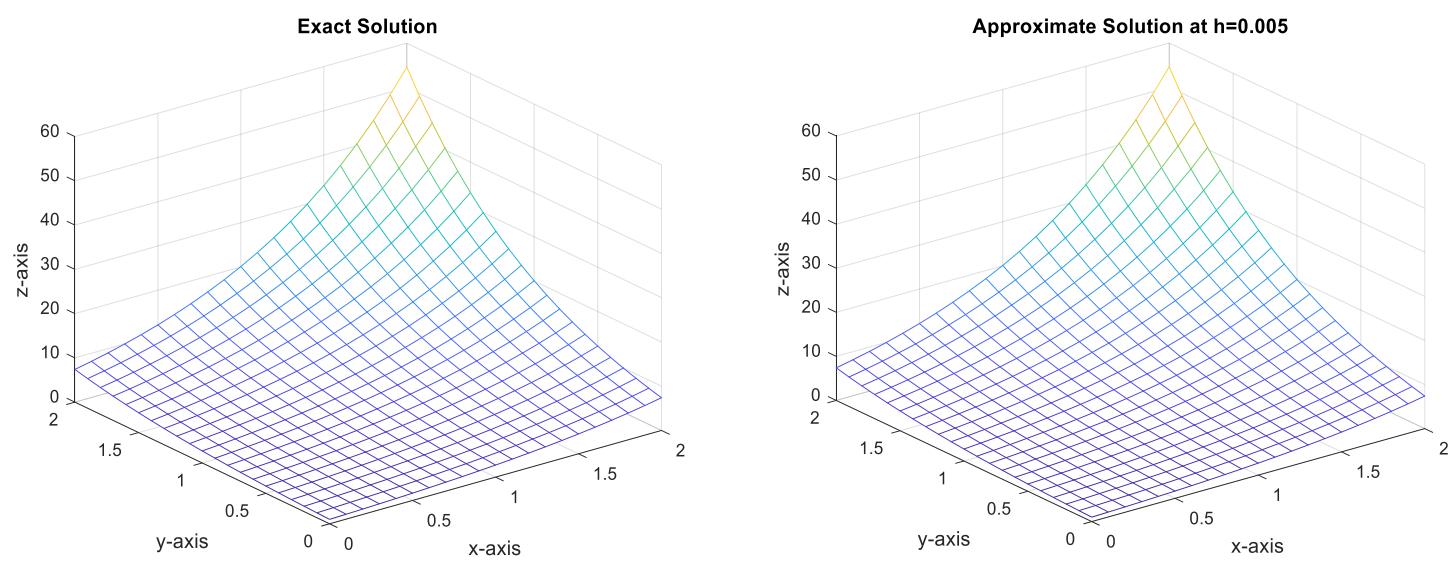

Figure 1. The graph of exact solution and approximate solution at $\mathrm{h}=0.005$ 
The graph of approximate solution at $\mathrm{h}=0.005$ is graphically similar to the graph of exact solution. Thus, we deduce that the relative errors of approximate solution are significantly small. Hence, both presentations for the approximate solution either in a tabular form or graphical illustration proved that the method is an effective method.

The fuzzy transform method is successfully applied to hyperbolic Goursat PDE where the numerical solution is accurate by comparing the relative error at different step size. The significant of fuzzy transform is it reduce the complexity of differential equation by transforming it to the algebraic equation. The proposed numerical method can be extend to differential equation involving more variables. The advantage of the proposed method is for every term in algebraic equation, it can be approximated by different existing approximation techniques and successively developed a new numerical method. Thus, the fuzzy transform method is a good numerical method for solving hyperbolic Goursat PDE.

\section{CONCLUSION}

In this paper, a new method has been successfully developed by utilizing the Fuzzy Transform for solving various engineering problem represent by hyperbolic Goursat partial differential equation. The fuzzy transform is utilized on both side of Goursat equation to produce a continuous approximation. Since various engineering problems involve complex model, the complexity can be reduced by applying fuzzy mathematics concept. The difficulties and complications of the proposed numerical method is overcome by developing a MATLAB program as well as with the aid of MAPLE software. Therefore, the computational numerical solution has been presented in this paper. Numerical methods are very significant to solve complex model considering the numerical methods can be effectively demonstrate in cases dealing with complex problems for which analytical solutions cannot be obtained or hand calculations cannot be made. Hence, this method can be an alternative numerical method to solve any physical problem represented by a hyperbolic Goursat PDE.

\section{REFERENCES}

[1] Day JT., "A Runge-Kutta Method for the Numerical Solution of the Goursat Problem in Hyperbolic Partial Differential Equation," Computer Journal, vol. 9(1), pp. 81-83, 1996.

[2] Taghvafard H. and Erjaee H. G., "Two-Dimensional Differential Transform Method for Solving Linear and NonLinear First problem," International Journal of Engineering and Mathematical Sciences, vol. 6(2), pp. 103-106, 2010.

[3] Pandey P. K., "A Finite Difference Method for Numerical Solution of Goursat Problem of Partial Differential Equation," Open Access Library Journal, vol. 1(06), p. 1, 2014.

[4] Wazwaz A. M., "On the Numerical Solution for the Goursat Problem," Applied Mathematics and Computation, vol. 59(1), pp. 89-95, 1993.

[5] Wazwaz A. M., "The Variational Iteration Method for a Reliable Treatment of Linear and Nonlinear Goursat Problem," Applied Mathematics and Computation, vol. 193(2) pp. 455-462, 2007.

[6] Ahmad J. and Mushtaq M., "Exact Solution of Linear and Non-linear Goursat Problems," Universal Journal of Computational Mathematic, vol. 3(1), pp. 14-17, 2015.

[7] Tuckwell H. C., "Numerical solutions of some hyperbolic stochastic partial differential equations with mixed derivatives including sine-Gordon equation," Cornell University Library. 2015.

[8] Perfilieva I., Fuzzy approach to solution of differential equations with imprecise data: application to reef growth problem. Academic Press, Amsterdam, pp. 275-300, 2003.

[9] Perfilieva I., Fuzzy Transforms. Rough and Fuzzy Reasoning: Rough versus Fuzzy. Springer-Verlag, 2004.

[10] Perfilieva I. and Vala'sek R., "Fuzzy Transforms in Removing Noise," Computational Intelligence, Theory and Applications (Advances in Soft Computing), Springer-Verlag, 2004.

[11] Perfilieva I. and Kreinovich V., "Fuzzy transforms of higher order approximate derivatives: A theorem," Fuzzy Sets and Systems, vol. 180(1), pp. 55-68, 2011.

[12] Perfilieva I., "Fuzzy transforms: Theory and applications," Fuzzy Sets and Systems, vol. 157(8), pp. 993-1023, 2006.

[13] Patanè G., "Fuzzy transform and least-squares approximation: Analogies, differences, and generalizations," Fuzzy Sets and Systems, vol. 180(1), pp. 41-54, 2011.

[14] Khastan A., Perfilieva I., and Alijani Z., "A new fuzzy approximation method to Cauchy problems by fuzzy transform," Fuzzy Sets and Systems, vol. 288, pp. 75-95, 2016.

[15] Coroianu L. and Stefanini L., "General approximation of fuzzy numbers by F-transform," Fuzzy Sets and Systems, vol. 288, pp. 46-74, 2016.

[16] Perfilieva I., Fuzzy transforms and universal approximation. EUSFLAT conference, pp. 529-533, 2003.

[17] Zadeh L., Fuzzy set theory. Information and Control, pp. 338-353, 1965.

[18] Wazwaz A. M., Partial Differential Equations and Solitary Waves Theory. Beijing: Higher of Education Press and Springer-Verlag Berlin Heidelberg. 2009.

[19] Wazwaz A. M., "The variational iteration method for a reliable treatment of the linear and the nonlinear Goursat problem," Applied Mathematics and Computation, vol. 193(2), pp. 455-462, 2007. 